\title{
Depression Therapy Using Chatbot
}

\author{
Arkodeep Biswas $^{1}$ | Ajay Kaushik ${ }^{2}$ \\ ${ }^{1}$ B. Tech Scholar, Department of Information Technology,Maharaja Agrasen Institute of Technology, Delhi, India \\ ${ }^{2}$ Assitant Professor, Department of Information Technology, Maharaja Agrasen Institute of Technology, Delhi, India
}

To Cite this Article

Arkodeep Biswas and Ajay Kaushik, "Depression Therapy Using Chatbot", International Journal for Modern Trends in Science and Technology, 6(12): 323-327, 2020.

\section{Article Info}

Received on 12-November-2020, Revised on 05-December-2020, Accepted on 11-December-2020, Published on 15-December-2020.

\section{ABSTRACT}

The objective of this paper is to build a Web Application based on Virtual voice and chat Assistant. The current study focuses on development of voice and text/chat bot specifically. It is specially being built for people who feel depressed and insists them to talk open mindedly which in turn pacifies them. As the name of the application suggests, App: An application to pacify people and make them as happy as a cat would be with his or her mother (the reason why a cat purrs). We will be using Dialog flow for the application design and Machine Learning as a part of Artificial Intelligence for Natural Language Processing (NLP), an easiest way to use Machine Learning libraries. At the back-end we will be using a database to store the communication history between the user and the bot. This application will only work on devices with Web operating system version-5.0 and above.

KEYWORDS: Artificial Intelligence, Virtual Assistant, Software Agent, Machine Learning, Natural LanguageProcessing

\section{INTRODUCTION}

Chatbot is a computer program which is designed to transcribe conversation with humans. It works on Artificial Intelligence. Chatbots are wontedly used in dialog systems (dialog systems or a conversational agent is a computer system which focuses on discourse with humans for purposes like customer service, providing basic information etc.). The term Chatbot is derived from Chatterbot, originally contrived by Michael Mauldin in 1994. A Virtual Assistant is a Software Agent that can perform tasks or services for an individual. Sometimes the term Chatbot is used as an alias to Virtual Assistants which are generally or specifically those accessed by online chat. Virtual Assistants does work through text, voice, and/or uploading images. Chatbots can be accessed through virtual assistants like Google assistant, Cortana, Siri etc., through messaging apps like
Facebook messenger etc., or through individual organizations' apps and websites. Chatbots are categorized into several categories such as conversational commerce which is an e-commerce via chat, analytics, communication, customer support, design, developer tools, education, entertainment, finance, food, games, health, Human Resources (HR), marketing, news, personal, productivity, shopping, social, sports, travel and utilities.

What we are working on will be specifically designed for interaction with the people who are depressed. It urges them to talk with their minds open. For instance, most people who are in depression tend to isolate themselves, they have trouble trusting people around them, they skip meals, and exaggerate situations they have no reason to worry about. The App we are working on helps them to get over all these challenging 
situations, App pacifies by drawing attention away from their worries. App also helps in their difficult situations by advising them appropriate ways to overcome difficulties.

\section{RELATED WORK}

MobileCoach, a mobile chat application related to human-bot interaction using text messages based on the concept of the user sending text messages to the bot which charges SMS cost to the person communicating with the bot. Additionally, the application is limited to text interaction with a predefined answer selection to the user; the user will be presented with a set of predicted response from which he is reckoned to choose one as a response to the bot. A mobile application employed with voice detector, ordinarily matched in cancer patients, with the use of prosodic and statistical traits extorted by voice signal for evoking depression. An online free service using a chatbot to motivate an individual in distress, experiencing hard times, serving with a theory of personalized chat that keeps a person involved in a conversation with the chatbot to negotiate ill-effects of depression. Woebot, an Artificial Intelligence therapy chatbot application which works on the principle of imminent text method; suggests appropriate responses based on the conversation between a man and the bot. MOST: Moderated online social therapy, a web application rendering assistance to those who are need of self-esteem, overcoming social anxiety, interacting with people, and recovering early psychosis. A chatbot for mental health counseling, intercommunication with the chatbot using emojis and GIFs while exploring for online help tips.

\section{PROPOSED WORK}

The concepts of papers published earlier recite on the notion of predictive text, imminent GIFs, and emojis, text messages with predefined answer selection, and voice detector matched in cancer patients. The objective of this paper is to formulate a theory related to a chatbot interaction with text and speech output, the responses will

be predicted based on a survey conducted by providing a questionnaire to the people who had suffered depression or other mental illness in the past. They are directed to answer a few questions regarding their journey of defeating their health condition. The questions also include their preference on how they desired to have interacted in the antiquity. Moreover, enquiring if they would be comfortable with either the predicted text suggestions or the transcribed text. Furthermore, whenever the person, who is expected to respond to the bot, does not acknowledge, consequently, the chatbot urges to respond recursively.

\subsection{ARCHITECTURE}

The figure Fig. 1, tells us about the architecture of the App. When the user registers himself into the application, they will be stored in the database, required when the bot greets the user. As soon as he concludes registering, the bot will prompt him to speak or type his response, interfaced through a microphone and keypad respectively to the application. Subsequently, the user's response will be processed with the help of Natural Language Processing followed by an algorithm implemented using Machine Learning. Finally, the output, obtained after classifying the text by correlating it with the previously existing data in the database, will be displayed to the user through the screen and the speaker as interfaces. FI

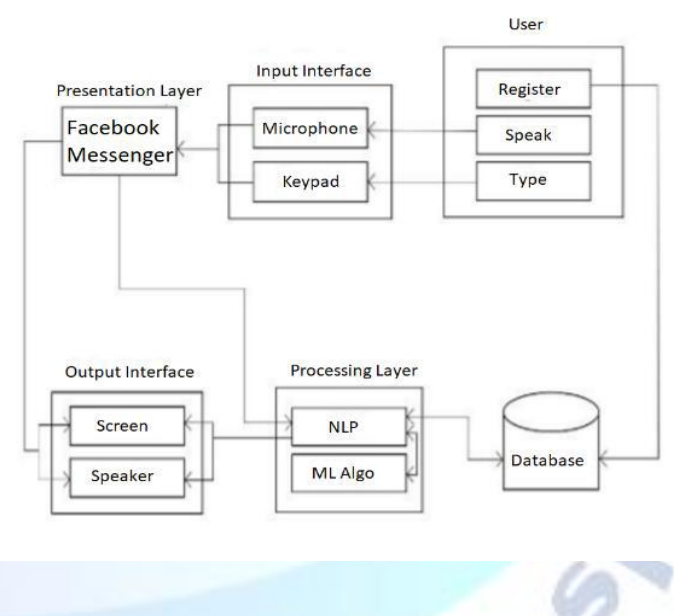

\subsection{WORKING ALGORITHM}

The figure Fig.2, is a simple working algorithm of the proposed study. The procedure starts as the user launches the application. The first and the foremost activity is a Splash Screen displaying the logo of App. Following the splash screen, application setup comes into the picture asking details of the user, which includes name and gender, the same will be stored in a database.

After setting up the application, the user will be shown the application interface where an acknowledgment message will be shown which is followed by waiting for the user's response. If the user does not respond then the bot repeatedly 
prompts the user to answer until he does When the user responds, Artificial Intelligence comes into the picture with the help of Machine Learning. As the Bot starts to converse with the user each response it gives is implemented using Machine Learning which is achieved through Python Language Python Language will be coupled with Dialogflow to achieve Natural Language Processing. Thereupon, the application continues to run until the user decides to quit.

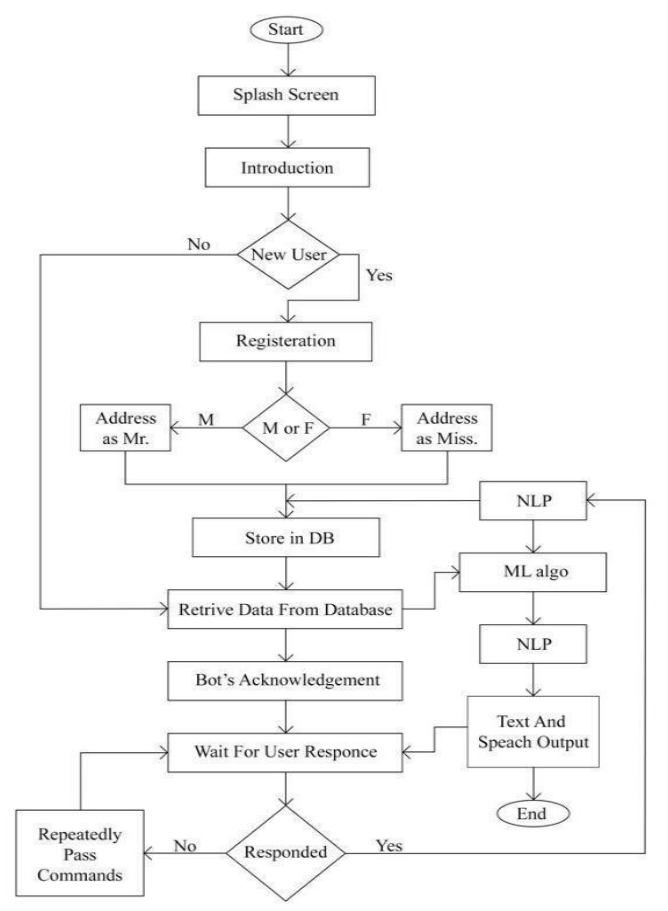

\subsection{MATHEMATICAL FORMULAE}

For a chatbot to be fully developed, both Machine Learning algorithms and Natural Language Processing are required.

Chatbots $=$ Machine Learning + Natural Language Processing. To build a chatbot, we have two crucial components to be supervised; Intent Classification, and Entity Recognition.

We use classification algorithms to achieve Intent Classification. Algorithms such as Support Vector Machine and Naive Bayes classifier are to be implemented. Rasa Natural Language Understanding can also be used for building an independent language parser and obtain Intent Classification Suppose a training dataset of $n$ support vectors of the form:

(X1,Y1), (X2,Y2), (X3,Y3)..., (Xn,Yn)

Yi represents the class that $\mathrm{Xi}$ belongs to. The principal objective of SVM is to identify a hyperplane that segregates two classes in a better way, has a maximum margin (the distance between hyperplane and data points). The former rule has the highest priority than

the latter. The linear equation for calculating parameters of SVM is For the first vector, $\Sigma$ aiSiS1 = $-1$

Similarly, the second vector, LaiSiS2 $=-1$

AS FOR THE NTH VECTOR, $\Sigma$ AISISN $=1$.

Here $a 1, a 2 \ldots$, an are the parameters and $S 1, S 2 \ldots$, $\mathrm{Sn}$ are the support vectors augmented with their respective bias values. Finally, we need to calculate the normal vector, Wi using the formula : W= $=$ aiSI Naive Bayes classification algorithm finds the probability of a given sample belonging to a particular class. It is based on the concept of Bayes theorem. Below is the formula for the same

$\mathrm{P}(\mathrm{c} / \mathrm{x})=(\mathrm{P}(\mathrm{x} / \mathrm{c})$ * $\mathrm{P}(\mathrm{c})) / \mathrm{P}(\mathrm{x})$

$\mathrm{P}(\mathrm{c} / \mathrm{x})$ and $\mathrm{P}(\mathrm{x} / \mathrm{c})$ are the conditional probabilities; the probability of class $\mathrm{x}$ having the attribute $\mathrm{c}$ and vice versa respectively. $\mathrm{P}(\mathrm{c})$ and $\mathrm{P}(\mathrm{x})$ are independent probabilities

\subsection{IMPLEMENTATION}

Beginning with the app design, the app design will be implemented using Dialogflow. Subsequently at the back-end a relational database, SQLite is used to store information in the form of tables as data under each attribute. SQLite is a default embedded software in Dialogflow. The Queries i.e., SQL statements, are written in Dialogflow through Java, a general-purpose programming language. The Queries in SQLite is equivalent to that of Oracles' MySQL, an open-source relational database management system. The database stores the information provided by the user during the application set-up. It contains two relational tables, one to store user information and the other to store data used for machine learning Now, as we have seen what happens in the front-end and at the back-end, it is time to present how the Machine Learning algorithm works. Let us take an illustration to explain this more lucidly and for a better understanding. For instance, when you have received an E-mail from a particular person in Gmail you must have noticed four suggestion as a response back to the sender, the question here is how do you think Gmail accurately predict responses relevant to the mail you received? -the answer is Machine Learning. You must have been wondering till now if Gmail could read you mind or might have found it wizardry. Now as the mystery is solved and out in the open its time for us to understand how exactly does Machine Learning 
work and how you yourself can apply it elsewhere which is what we are doing. In this study we will use Machine Learning to predict the user's response to the text sent by the bot and vice versa. Now, we shall see how the expected output looks like. As described earlier, as the user launches the application, the Splash screen is displayed as shown in Fig.3.Subsequently, as shown in the Fig.4, the Application description will be displayed where the user is asked to read. But it is not mandatory for the user to read it wholly, if the user wishes to continue without reading, he may skip the introduction part and continue with the application set up. The introductory part says "This is a brief introduction of the application and we suggest you read this before continuing but if you are in a hurry and wish to continue without reading this, find and click on the "Continue" button below. Followed by the application setup where the personal details such as name and gender of the user will be asked to be filled. Soon after entering the details the user is supposed to tap continue where he will be taken to the next activity i.e., main activity, where the user will be able to interact with the bot.

\section{RESULTS}

Current results show accuracy of $84.14 \%$ on the training data,

accuracy of $83.32 \%$ on the cross validation data and accuracy of

$60.17 \%$ on the test data.

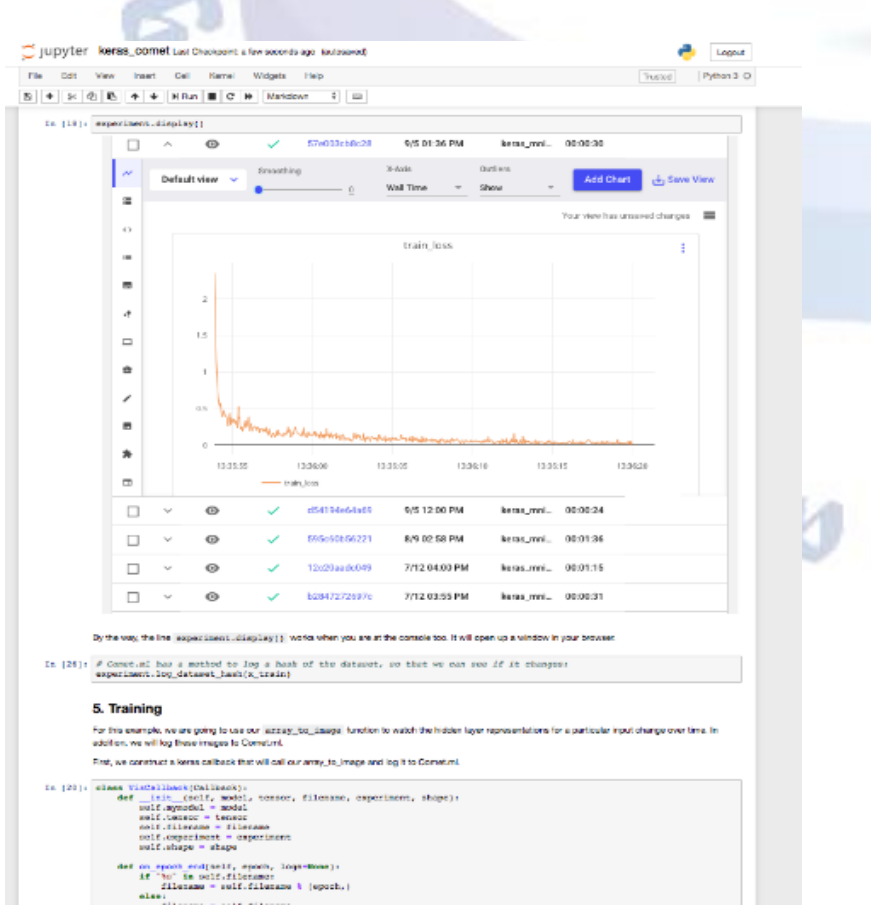

Fig 3 : Result(1)

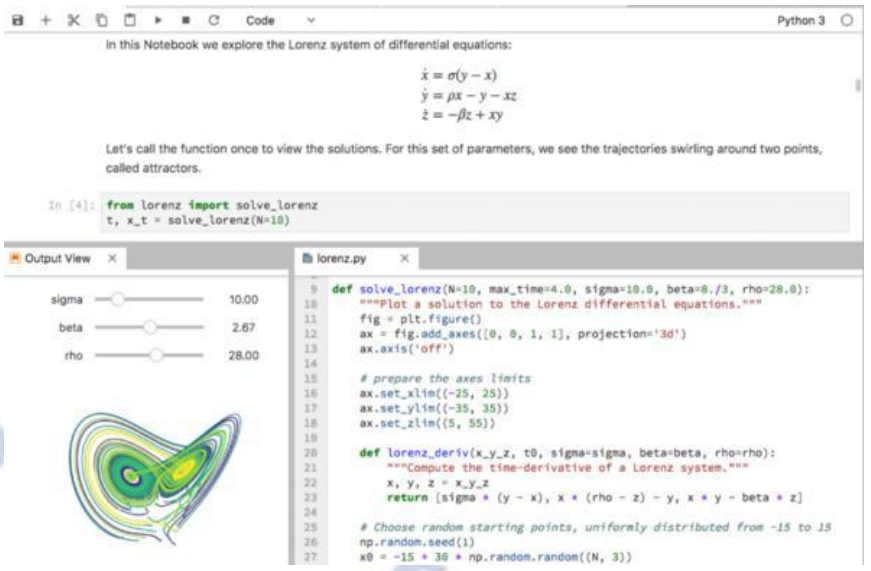

Fig 4 : Result (2)

\subsection{FUTURE DEVELOPMENTS}

As we could only reach satisfactory level of the study due to the time constraint, further advancement is expected to be carried out in the future. For instance, we are planning to summate a facial recognition feature which gives the application an upgrade. This feature gives helps the user when he is not inclined to talk or converse with the bot. The bot waits for a couple of minutes or so for the users' response, if the bot does not get any response then it automatically detects the face of the user and acts relevant to the expressions of the person i.e., if the person feels down then the bot plays fun music or shows playful videos of pets. Additionally, if the person is bored, we are planning to add a game option to kill the boredom. Furthermore, during setup the person will be given an option to enter their medical details which includes medicine dosage and timings with which daily reminder will be enabled for medicines intake. There is also an idea to implement alarms to setup automatically with respect to their bedtime and wakeup time. As described in the introduction this application is mainly for depressed people, but in future we are planning to make this application Autistic friendly, and also for people suffering with Schizophrenia. As we all know that autistic people have difficult time in connecting with people, so App will help them to work on it by assuring them that they are not alone in this world and helps them share their thoughts. As for Schizophrenic people, when they feel threatened by situations that are happening around them, App assures them that they are living in an illusion and helps them to come out of it by explaining them what exactly is happening to them, by playing pleasant music and make them realize that there is nothing 
to be worried about. As described in the introduction this application is mainly for depressed people, but in future we are planning to make this application Autistic friendly, and also for people suffering with Schizophrenia. As we all know that autistic people have difficult time in connecting with people, so App will help them to work on it by assuring them that they are not alone in this world and helps them share their thoughts. As for Schizophrenic people, when they feel threatened by situations that are happening around them, App assures them that they are living in an illusion and helps them to come out of it by explaining them what exactly is happening to them, by playing pleasant music and make them realize that there is nothing to be worried about. We are also planning to add a signup option for App, where users will be able to back up their account. Users will be given an option to back up their account to their Google account or Microsoft account or Facebook account.

\section{CONCLUSION}

Hence, we conclude that the App, a chatbot application that is being built is used for interaction for depressed people. It will be there in a persons' good and bad times. It urges them to talk with their minds open which helps them to start opening up about their feelings. App can also be used by non-depressed people but it is more beneficial to those who are suffering with depression. As the study is in progress, we would like to conclude further by taking surveys from people surrounding us.
7. Simon Hoermann, Kathryn L McCabe, David N Milne, et al. Application of Synchronous Text-Based Dialogue Systems in Mental Health Interventions: Systematic Review.

8. Robert R Morris, Kareem Kouddous, Rohan Kshirsagar, et al. Towards an Artificially Empathic Conversational Agent for Mental Health Applications: System Design and User Perceptions.

9. Kathleen Kara Fitzpatrick, Alison Darcy, Molly Vierhile. Delivering Cognitive Behavior Therapy to Young Adults With Symptoms of Depression and Anxiety Using a Fully Automated Conversational Agent (Woebot): A Randomized Controlled Trial.

\section{REFERENCES}

1. Tobias Kowatsch, Dirk Volland, Iris Shih, et al. Design and Evaluation of a Mobile Chat App for the Open Source Behavioral Health Intervention Platform MobileCoach

2. Alexandros Ronioti, Manolis Tsiknakis. Detecting Depression Using Voice Signal Extracted by Chatbots: A Feasibility Study

3. Pratik Kataria, Kiran Rode, Akshay Jain User Adaptive Chatbot for Mitigating Depression

4. Alison Darcy, Andrew Ng, Athena Robinson et al. Woebot

5. Simon D'Alfonso, Olga Santesteban-Echarri, Simon Rice et al. Artificial Intelligence-Assisted Online Social Therapy for Youth Mental Health.

6. Gillian Cameron, David Cameron, Gavin Megaw et al. Towards a chatbot for digital counselling 